\title{
RBS/Channeling and TEM Study of Damage Buildup in Ion Bombarded GaN
}

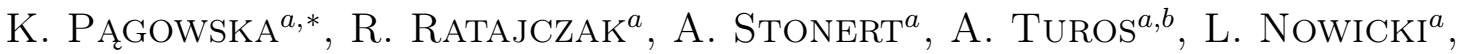 \\ N. SAThish ${ }^{a, b}$, P. JóŹWIK ${ }^{a, b}$ ANd A. MUECKLiCH ${ }^{c}$ \\ ${ }^{a}$ Sołtan Institute for Nuclear Studies, 05-400 Otwock/Świerk, Poland \\ ${ }^{b}$ Institute of Electronic Materials Technology, Wólczyńska 133, 01-919 Warsaw, Poland \\ ${ }^{c}$ Helmholtz-Zentrum Dresden-Rossendorf, POB 510119, D-01314 Dresden, Germany
}

\begin{abstract}
A systematic study on structural defect buildup in $320 \mathrm{keV}$ Ar-ion bombarded GaN epitaxial layers has been reported, by varying ion fluences ranged from $5 \times 10^{12}$ to $1 \times 10^{17}$ at. $/ \mathrm{cm}^{2}$. $1 \mu \mathrm{m}$ thick GaN epitaxial layers were grown on sapphire substrates using the metal-organic vapor phase epitaxy technique. Rutherford backscattering/channeling with $1.7 \mathrm{MeV}^{4} \mathrm{He}$ beam was applied for analysis. As a complementary method high resolution transmission electron microscopy has been used. The later has revealed the presence of extended defects like dislocations, faulted loops and stacking faults. New version of the Monte Carlo simulation code McChasy has been developed that makes it possible to analyze such defects on the basis of the bent channel model. Damage accumulation curves for two distinct types of defects, i.e. randomly displaced atoms and extended defects (i.e. bent channel) have been determined. They were evaluated in the frame of the multistep damage accumulation model, allowing numerical parameterization of defect transformations occurring upon ion bombardment. Displaced atoms buildup is a three-step process for GaN, whereas extended defect buildup is always a two-step process.
\end{abstract}

PACS: 61.82.Fk, 61.85.+p, 68.55.Ln, 68.35.Dv

\section{Introduction}

Gallium nitride plays an important role in short wavelength optoelectronics and high-power, high frequency microelectronics. Lack of commercially available free standing GaN substrates makes it necessary to use alternative ones such as sapphire $\left(\mathrm{Al}_{2} \mathrm{O}_{3}\right)$. Ion implantation proved to be an efficient way for doping of this semiconductor compound and as a consequence provided a very strong motivation for studies on defect formation and their transformations.

In our contribution to the previous Ion Conference preliminary results on the defect buildup in GaN, AlGaN and AlN upon $320 \mathrm{keV}$ Ar-ion bombardment has been reported [1]. The study was exclusively based on the Rutherford backscattering/channeling (RBS/C) experiments evaluated by means of the Monte Carlo simulation code McChasy [2]. The version of the McChasy code available at that time allowed only calculating defects in the form of randomly displaced atoms (RDA). As demonstrated in Ref. [3] such an analysis cannot provide reliable data. A new approach to defect analysis in ion implanted GaN epitaxial layers has been applied. The modified Monte Carlo code McChasy makes use of the bent channel (BC) model described in detail in Ref. [3]. Such an approach makes it possible to separate effects due to the scattering by RDA and BC defects and forms the basis for their quantification.

\footnotetext{
* corresponding author; e-mail: karolina.pagowska@gmail.com
}

When applied to ion implanted GaN, the BC model has revealed a large component of $\mathrm{BC}$ defects that was clearly present for all implantation fluences applied. In order to determine the detailed type of the $\mathrm{BC}$ defects high resolution transmission electron microscopy (HRTEM) has been used as a complementary method. Precise visualization of such defects allowed their parameterization thus, creating the basis for reliable Monte Carlo simulations of RBS/C spectra.

In this paper we report on the new evaluation of channeling analysis of Ar-ion implanted GaN revealing the mechanism of defect transformations and eventually the precise morphology of studied epitaxial layers.

\section{Experimental}

GaN layers were grown on sapphire substrates in AIX 200/4 RF-S metal organic vapor phase epitaxy low-pressure reactor. The source gases were trimethylgallium (TMGa), and ammonia $\left(\mathrm{NH}_{3}\right)$. High purity hydrogen was used as a carrier gas. A standard two-step deposition process was employed $[4,5]$. First AlN nucleation layer (NL) of approximately $20 \mathrm{~nm}$ thickness was deposited at $550^{\circ} \mathrm{C}$ and followed by GaN layer deposition at $1140{ }^{\circ} \mathrm{C}$.

Implantation with $320 \mathrm{keV}$ Ar ions to fluence ranging from $5 \times 10^{12}$ to $1 \times 10^{17}$ at. $/ \mathrm{cm}^{2}$ was performed at room temperature (RT) in the Institute of Electronic Materials Technology. In order to avoid the sample heating the implantation current density was kept below $1 \mu \mathrm{A} / \mathrm{cm}^{2}$. RBS/C and HRTEM measurements were performed at the Institute for Ion Beam Physics and Ma- 
terials Research Forschungszentrum Dresden, Germany. Virgin and implanted samples were analyzed with RBS/ channeling using $1.7 \mathrm{MeV}{ }^{4} \mathrm{He}$-ions. Channeling spectra along [0001] axis measured for virgin samples revealed a very low channeling minimum yield, $\chi_{\min } \leq 2 \%$. High-resolution cross-sectional transmission electron microscopy investigations have been performed using a Ti$\tan 80-300$ facility manufactured by FEI. The microscope is equipped with an image corrector, which strongly reduces any delocalization yielding the point resolution of about $0.1 \mathrm{~nm}$.

\section{Results and discussion}

Two regions can be distinguished in the aligned channeling spectra shown in Fig. 1. The first one is delimited by pronounced peaks located in the vicinity of GaN/sapphire interface (around channel \# 200-300) and is due to defect agglomeration generated by layer/substrate lattice misfit and remnants of polycrystalline NL. The second region above channel \# 600, reflects the top part of GaN layer of approximately $600 \mathrm{~nm}$ thickness. With increasing Ar-ion fluence scattering yield in this region also increases indicating continuous defect buildup.

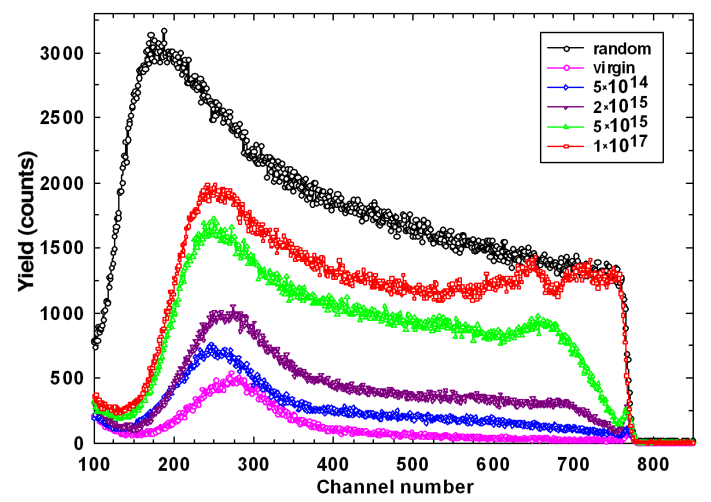

Fig. 1. Random and [0001] aligned RBS spectra measured at $1.7 \mathrm{MeV}$ incident ${ }^{4} \mathrm{He}$ ion energy for $1160 \mathrm{~nm}$ thick GaN epilayer implanted to different fluences of $320 \mathrm{keV}$ Ar ions.

In Ref. [1] three step damage accumulation in GaN upon Ar-ion bombardment has been reported. Consequently, three regions can be distinguished: low fluence - below $2 \times 10^{15} \mathrm{Ar} / \mathrm{cm}^{2}$, medium fluence - below $5 \times 10^{16} \mathrm{Ar} / \mathrm{cm}^{2}$, high fluence - above $5 \times 10^{16} \mathrm{Ar} / \mathrm{cm}^{2}$.

To get detailed insight into structural transformations HRTEM analysis has been performed. Figure 2 shows TEM micrograph for GaN sample bombarded to the fluence of $5 \times 10^{14} \mathrm{Ar} / \mathrm{cm}^{2}$. Low density of defects, predominantly stacking faults are observed. Increase of ion fluence above the critical fluence of "stage I-stage II" transition (i.e. $2 \times 10^{15} \mathrm{Ar} / \mathrm{cm}^{2}$ ) produces totally different structure. As can be seen in Fig. 3 the increasing density of stacking faults led to the formation of quite a large density of dislocation that coalesce with increasing ion fluence. Upon bombardment to fluences above $5 \times 10^{16} \mathrm{Ar} / \mathrm{cm}^{2}$ (i.e. stage II-stage III transition), another structural transformation occurs. Complicated multilayer structure develops as shown in Fig. 4. On the top an amorphous layer containing some gas bubbles has developed, beneath is a layer containing high density dislocations as in stage II. Next deepest layer is again completely amorphized, the observed bubbles are apparently Ar bubbles. The later assumption corresponds well with the lowering of scattering yield in the vicinity of channel 680 in the spectrum for the highest fluence in Fig. 1. The deepest damaged layer contains again extended and planar defect structure, which is the remnant of stage II.

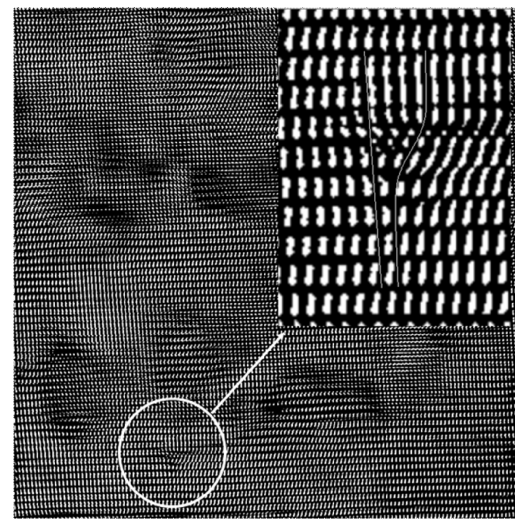

Fig. 2. TEM micrograph for GaN epilayer implanted with $5 \times 10^{14} \mathrm{Ar} / \mathrm{cm}^{2}$. Magnification $620 \mathrm{k} \times$.

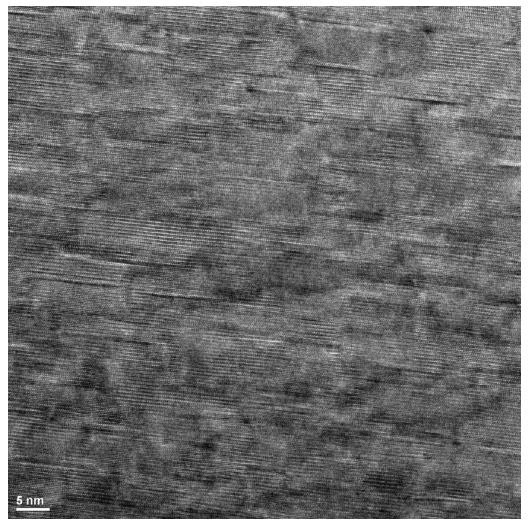

Fig. 3. TEM micrograph for GaN epilayer implanted with $5 \times 10^{15} \mathrm{Ar} / \mathrm{cm}^{2}$. Magnification $300 \mathrm{k} \times$.

Following the TEM observations the RBS/C spectra of Fig. 1 have been simulated using the modified McChasy code. First important finding was that independently of the bent channel parameters none of these spectra can be reproduced using BCs only. It was necessary to add an important amount of RDAs to accomplish this task. As a consequence, one has to admit that besides of the large amount of dislocations substantial concentration of displaced atoms, vacancies and irregu- 


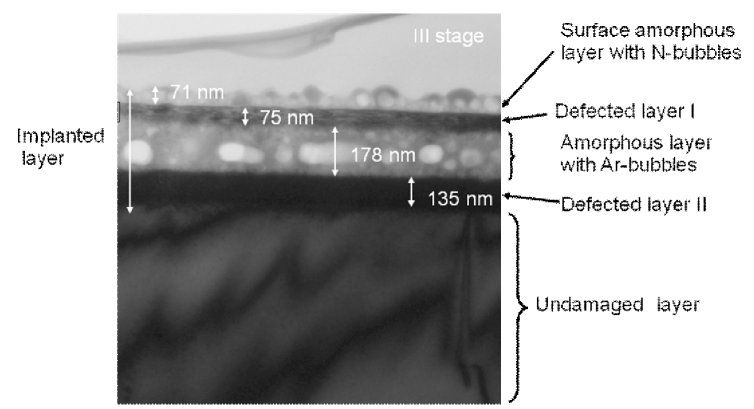

Fig. 4. TEM micrograph for GaN epilayer implanted with $1 \times 10^{17} \mathrm{Ar} / \mathrm{cm}^{2}$. Magnification $4 \mathrm{k} \times$.

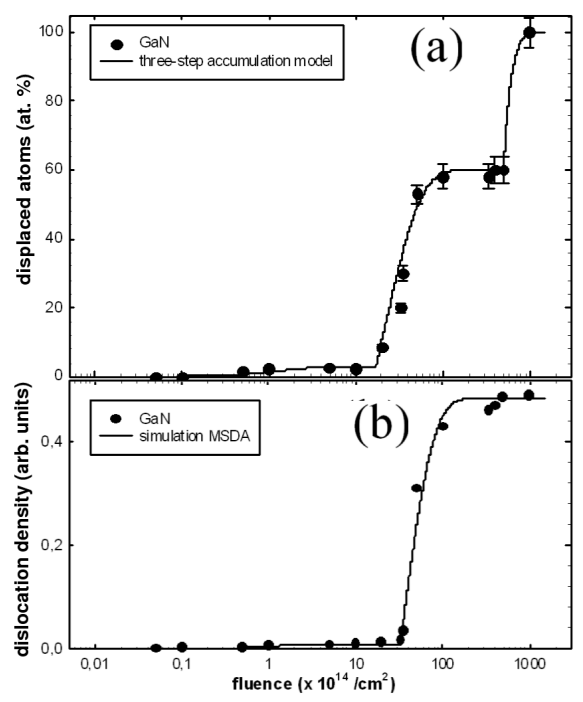

Fig. 5. Damage accumulation curves for randomly displaced atoms RDA (a) and BC defects (b).

lar defect clusters has been produced. They cannot be visualized by TEM.

Results of RBS/C measurements are summarized in Fig. 5a and b. Defect accumulation curve for RDA evolves through three stages and is in accordance with Ref. [1] and the other literature data [6-10]. Inclusion of $\mathrm{BC}$ defects in our simulations led to reduction of stage II level by $15 \%$. On the other hand, accumulation of BC defects exhibits two stages only. Since stage III leads to amorphization of substantial part of implanted layer, further increase of dislocation concentration cannot be expected. However, one can see that in stage III, the damaged layer I (see Fig. 4) dislocation concentration, $V$, is lower as compared to the samples of stage II. The fact that this is not the case can be explained by the close examination of Fig. 4. Beyond the second amorphous layer there is still a deepest region (i.e. damaged layer II in Fig. 4) containing defect structures characteristic for stage II. They have been produced by the deep tail of incident ions depth distribution that plays an especially important role at high fluences.

\section{Conclusions}

Presented results allow us to draw the final conclusions on the nature of damage buildup in ion bombarded GaN. According to our model, defect structure evolution is driven by well established thermodynamical factors.

In stage I, vacancies and interstitial defect agglomeration leads to formation of defects with low scattering cross-section for channeled ions. TEM micrographs indicate that these defects are faulted loops and stacking faults. Our X-ray diffraction (XRD) measurements [11] have shown that in stage I, a continuous tensile stress builds up along the $c$-axis with increasing fluence and is the driving force for transition into stage II.

Coalescence of variety of dislocation takes place in stage II. With prolonged ion bombardment, dislocations grow by trapping of simple defects created by successive collision cascades. In parallel, at given fluence, growing impurity concentration attains the level at which formation of gas bubbles becomes possible. Trapping of defects by these bubbles becomes efficient enough to preclude formation of dislocations and loops. Moreover, dislocation disrupted by consecutive collision cascades cannot reconstruct because mobile defects are preferentially trapped by bubbles. Hence, bubble formation is the most probable driving force for transition to stage III.

\section{References}

[1] K. Pagowska, R. Ratajczak, A. Stonert, L. Nowicki, A. Turos, Vacuum 83, S145 (2009).

[2] L. Nowicki, A. Turos, R. Ratajczak, A. Stonert, F. Garrido, Nucl. Instrum. Methods Phys. Res. B 240, 277 (2005).

[3] A. Turos, L. Nowicki, A. Stonert, K. Pągowska, J. Jagielski, A. Muecklich, Nucl. Instrum. Methods Phys. Res. B 268, 1718 (2010).

[4] X.H. Wu, D. Kapolnek, E.J. Tarsa, B. Heying, S. Keller, B.P. Keller, U.K. Mishra, S.P. DenBaars, J.S. Speck, Appl. Phys. Lett. 68, 1371 (1996).

[5] M. Kamp, Opt. Quantum Electron. 32, 227 (2000).

[6] S.O. Kucheyev, J.S. Williams, C. Jagadish, Vacuum 73, 93 (2004)

[7] S.O. Kucheyev, J.S. Williams, S.J. Pearton, Mater. Sci. Eng. 33, 51 (2001).

[8] W. Jiang, W.J. Weber, C.M. Wang, Nucl. Instrum. Methods Phys. Res. B 206, 1037 (2003).

[9] C. Liu, A. Wenzel, J.W. Gerlach, X.F. Fan, B. Rauschenbach, Surf. Coat. Technol. 128, 455 (2000).

[10] E. Wendler, A. Kamarou, E. Alves, K. Gartner, W. Wesch, Nucl. Instrum. Methods Phys. Res. B 206, 1028 (2003).

[11] E. Wierzbicka, M. Wójcik, J. Gaca, K. Pągowska, A. Turos, to be published. 